\title{
The correction of the relapsed club foot by closed distraction
}

\author{
Said Saghieh • Abdo Bashoura • Ghina Berjawi • \\ Nadim Afeiche $\cdot$ Rayan Elkattah
}

Received: 28 April 2009/ Accepted: 14 September 2010/Published online: 1 October 2010

(C) The Author(s) 2010. This article is published with open access at Springerlink.com

\begin{abstract}
Correction of a relapsed clubfoot deformity by distraction with an external fixator is a recognized alternative to open surgery. Most published series report a good outcome but none are prospective observational studies using the scoring system of the International Clubfoot Study Group (ICFSG). We present a series of 9 relapsed club feet treated with closed gradual distraction using this scoring method.
\end{abstract}

Keywords External fixator - Club foot - Ilizarov technique

\section{Introduction}

Club foot occurs in approximately 1 in 1,000 live births. Surgical correction through variants of the posteromedial release has been reasonably successful in restoring a normal shape to the foot. However, $20 \%$ of these cases will relapse [1]. The optimum management for these relapses is uncertain. Although the treatment method and outcome will depend on the severity and the type of deformity, open surgery with soft-tissue releases (with or without bone osteotomies) is associated with wound complications and an increase in stiffness of the ankle and foot joints.

Correction of a relapsed deformity by distraction with an external fixator is a recognized alternative. Most published

S. Saghieh $(\bowtie) \cdot$ A. Bashoura $\cdot$ N. Afeiche

Surgery Department, American University of Beirut,

AUB_Medical Center, Cairo street, Beirut, Lebanon

e-mail: ss15@aub.edu.lb

G. Berjawi · R. Elkattah

Diagnostic Radiology Department,

American University of Beirut, Beirut, Lebanon series report a good outcome but none are prospective observational studies using the scoring system of the International Clubfoot Study Group (ICFSG).

We present a series of 9 relapsed club feet treated with closed gradual distraction using this scoring method.

\section{Materials and methods}

Between July 2002 and April 2008, there were eight patients (five boys and three girls) who presented with a relapsed club foot. One boy had arthrogryposis and presented with bilateral relapsed club feet. The mean age was 5.6 years (3-9). All children had at least one open surgery with relapse. One patient had 2 previous posteromedial releases performed. The patients were evaluated preoperatively using clinical and radiographic methods of the ICFSG [2].

Surgery was performed under general anaesthesia. The circular frame was assembled in the operating room in a standardized fashion for all patients. The frame was applied to simulate the deformity. It consists of a tibial assembly comprising 2 rings connected to the bone by 3 half-pins or 2 wires and 2 half-pins. The calcaneal half-ring was fixed with 2 olive wires $(1.5 \mathrm{~mm})$, and the forefoot half-ring was fixed to the metatarsals by 2 wires $(1.5 \mathrm{~mm})$. The rings were connected with hinges and threaded rods that allowed correction of the various components of the deformity. A talar wire was not used but, in one patient, an epiphyseal wire in the distal tibia was inserted to prevent epiphysiolysis.

The patients spent one night in hospital before being discharged home. After 5 days, the patients were seen in the clinic and distraction begun. All of the deformities were simultaneously corrected. The adductus and cavus deformities were corrected by differential lengthening of the 
medial and lateral rods connecting the hindfoot and the forefoot half-rings at a rate of 3 and $1 \mathrm{~mm} / \mathrm{day}$, respectively. The hindfoot equinovarus was also corrected with differential lengthening of the 3 rods which connect the tibial assembly to the calcaneal half-ring. The distraction rates were, from medial to posterior to lateral, 3, 2 and $1 \mathrm{~mm}$ per day, respectively. An anterior rod helped with the correction of equinus through gradual shortening at a rate equal to double the rate of distraction of the posterior middle rod on the calcaneal half-ring.

The patients were seen in clinic weekly during the distraction period and then at every 3 weeks. Distraction was continued until a neutral ankle position or slight overcorrection with $5-10^{\circ}$ of dorsiflexion, $10^{\circ}$ of heel valgus and a straight lateral border of the foot had been obtained. Towards the end of the correction, the anterior rod was exchanged with 2 rods to correct any residual supination. Radiographs were requested after full correction.

A combination of non-steroidal anti-inflammatory medication (ibuprofen) and an analgesic (tramadol hydrochloride) was used for pain control throughout the correction phase in all children. After frame removal under general anaesthesia, a below knee cast was applied for 2-4 weeks, followed by an ankle foot orthosis for night time splinting for 6 months. The patient was seen at 6 weeks, 3 and 6 months and then two-yearly. At the latest follow-up, radiograms of the ankle and foot were performed, and the ICFSG score was recalculated.

The details of the 9 treated feet, the period in external fixation, the follow-up interval and both the preoperative and the latest ICFSG scores are presented in Table 1. These results were assessed using the Wilcoxon signed-rank test (SPSS 11.5.1 for Windows). $P$ values of 0.05 or less were considered significant.

The ICFSG scoring system is based on 3 criteria, each with a list of measurements. The preoperative and postoperative values of different components of ICFSG score system were listed in 3 tables delineating the morphology (Table 2), the functional evaluation (Table 3) and the radiologic evaluation (Table 4). The total score ranges from 0 to 60 points. Categorization of scores follows: $0-5$ points as an 'excellent' outcome; 5-15 points as a 'good' outcome; $16-30$ points as a 'fair' outcome; and more than 30 points as a 'poor' outcome.

\section{Results}

The median follow-up was 3.5 years $(0.5-7.5)$. Eight feet were graded as poor and one as fair at presentation. At the latest follow-up, the ICFSG score was excellent in 3 feet, good in 4 and fair in 2 feet. The median preoperative ICFSG score was 40 (26-45) and at the latest follow-up 7 (5-30). This difference was statistically significant with $P$ value of 0.0039 using the Wilcoxon signed-rank test. A plantigrade foot was accomplished in all patients at the end of the treatment. However, at the latest follow-up, there was a relapse of the equinovarus deformity in two feet.

Figures 1 and 2 showed two patients with club feet before, during and after the treatment by closed distraction. Table 2 describes the improvement in foot morphology. The median preoperative and postoperative morphology scores were 9 (7-11) and $1(0-6)$, respectively. The functional evaluation scores are shown in Table 3 . The median score was 18 (12-23) preoperatively and became 4 (3-16) at the latest follow-up. The radiographic score is summarized in Table 4 . The median score was 11 preoperatively (7-12) and 2 at the latest follow-up (1-8).

We encountered several complications during the treatment, most of which were related to superficial pin-site infections. The latter occurred in all patients and were successfully treated with oral antibiotics. Anterior subluxation of the talus occurred in two patients. They were treated with modification of the frame in the clinic to allow gradual posterior translation. Severe flexion contracture of the toes developed in 4 patients. They were managed by adding a foot plate and elastic toe suspenders during the treatment phase. These contractures resolved after gentle
Table 1 Details of the 8 feet and ICFSG score outcomes

\begin{tabular}{lllllllc}
\hline Case & Sex & $\begin{array}{l}\text { Age at } \\
\text { surgery (years) }\end{array}$ & Side & $\begin{array}{l}\text { Duration } \\
\text { follow-up (years) }\end{array}$ & $\begin{array}{l}\text { Time in } \\
\text { frame (days) }\end{array}$ & $\begin{array}{l}\text { Preop } \\
\text { ICFSG score }\end{array}$ & $\begin{array}{l}\text { Postop } \\
\text { ICFSG score }\end{array}$ \\
\hline 1 & F & 8 & L & 7 & 130 & 42 & 5 \\
2 & M & 6 & L & 6 & 75 & 34 & 5 \\
3 & M & 9 & L & 5 & 90 & 40 & 27 \\
4 & M & 3 & R & 3 & 50 & 26 & 6 \\
$5^{\text {a }}$ & M & 4 & R & 3 & 100 & 43 & 31 \\
$6^{\text {a }}$ & M & 5 & L & 2 & 80 & 40 & 15 \\
7 & F & 5 & L & 2 & 75 & 31 & 5 \\
8 & M & 5 & L & 1 & 90 & 34 & 7 \\
9 & F & 9 & L & 0.5 & 90 & 45 & 7 \\
\hline
\end{tabular}

${ }^{\text {a }}$ Bilateral 


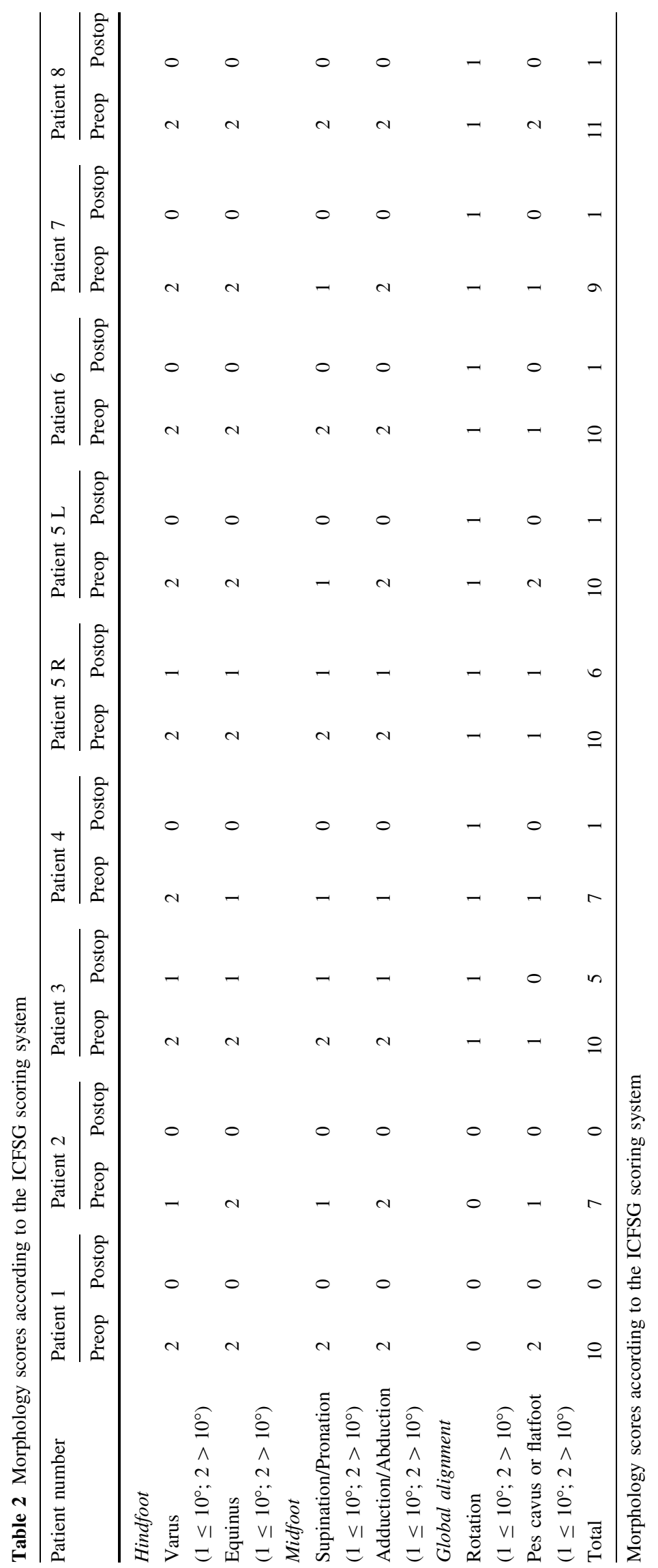




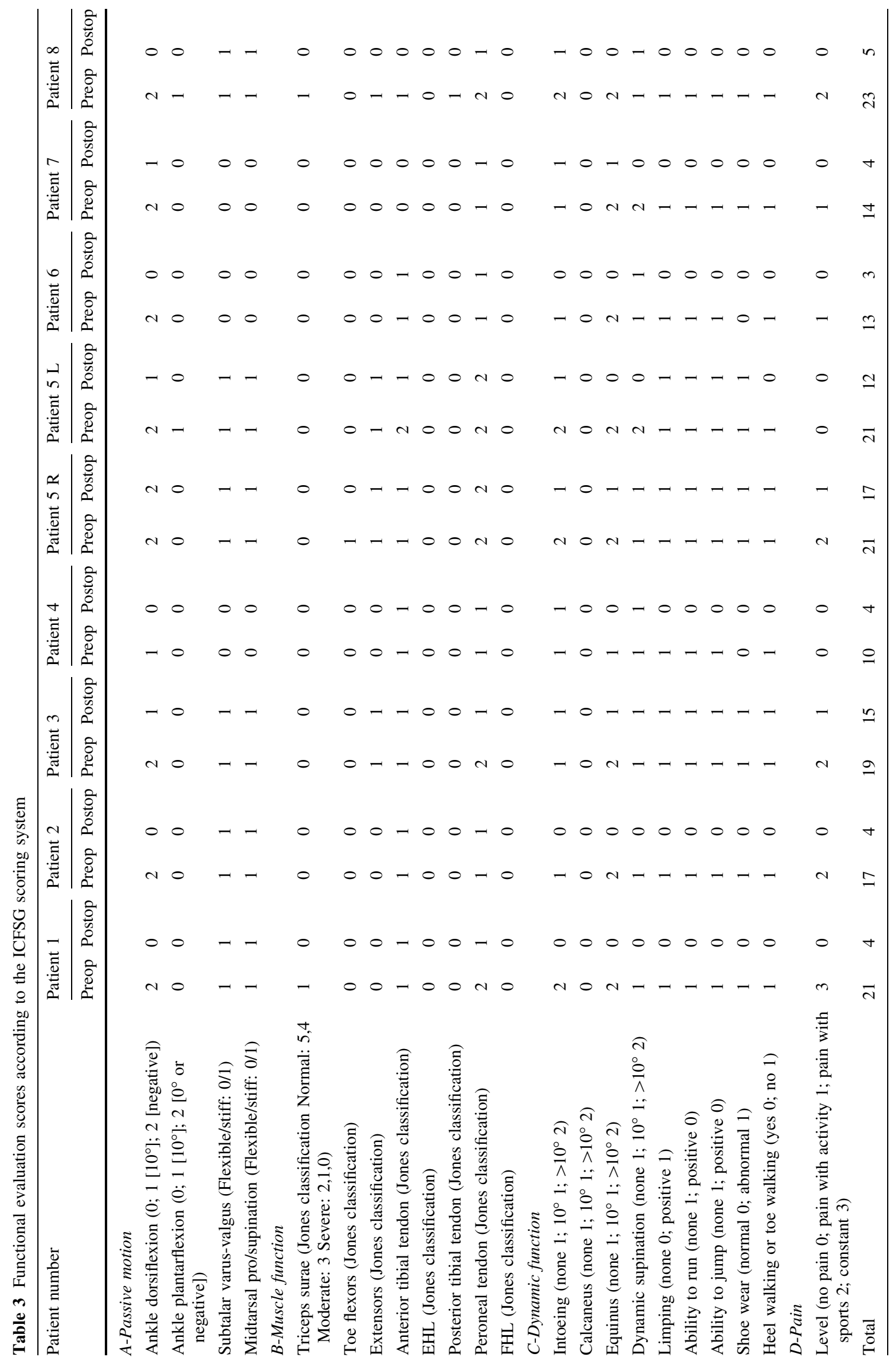




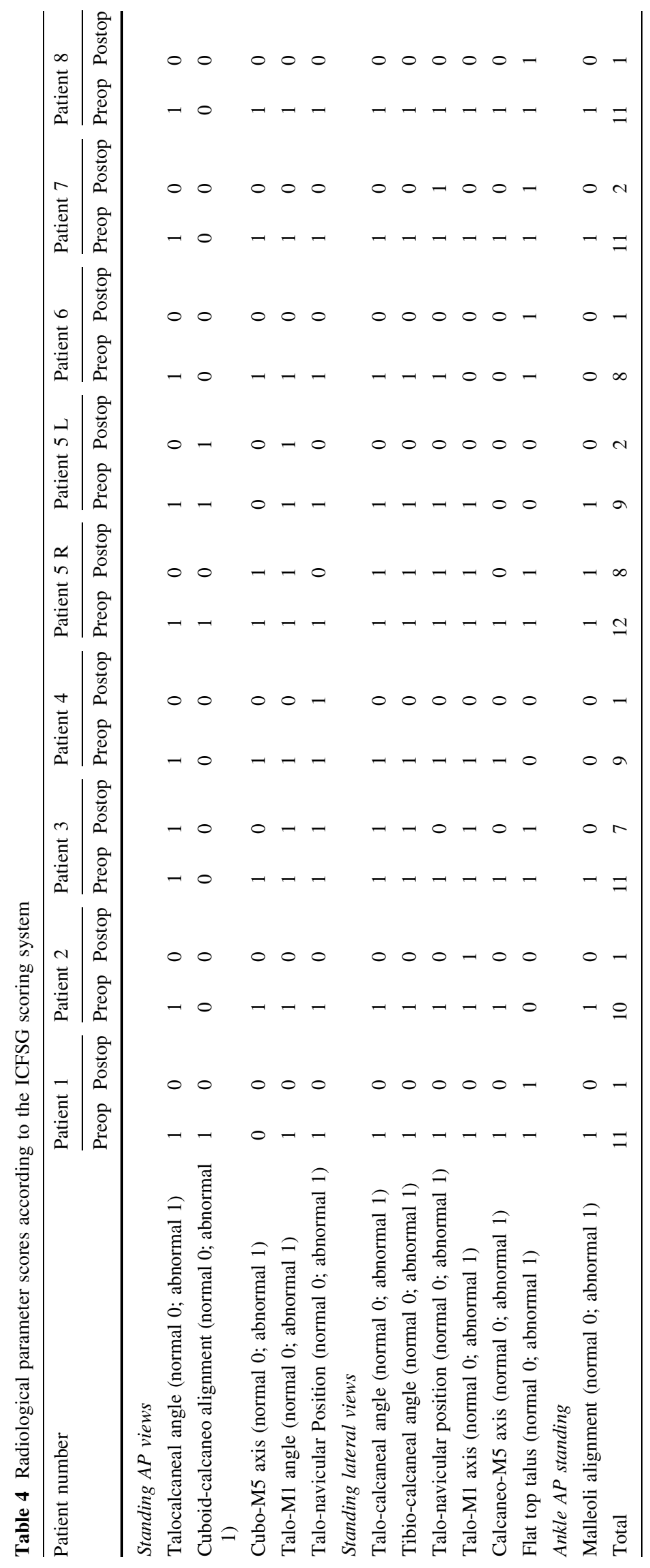



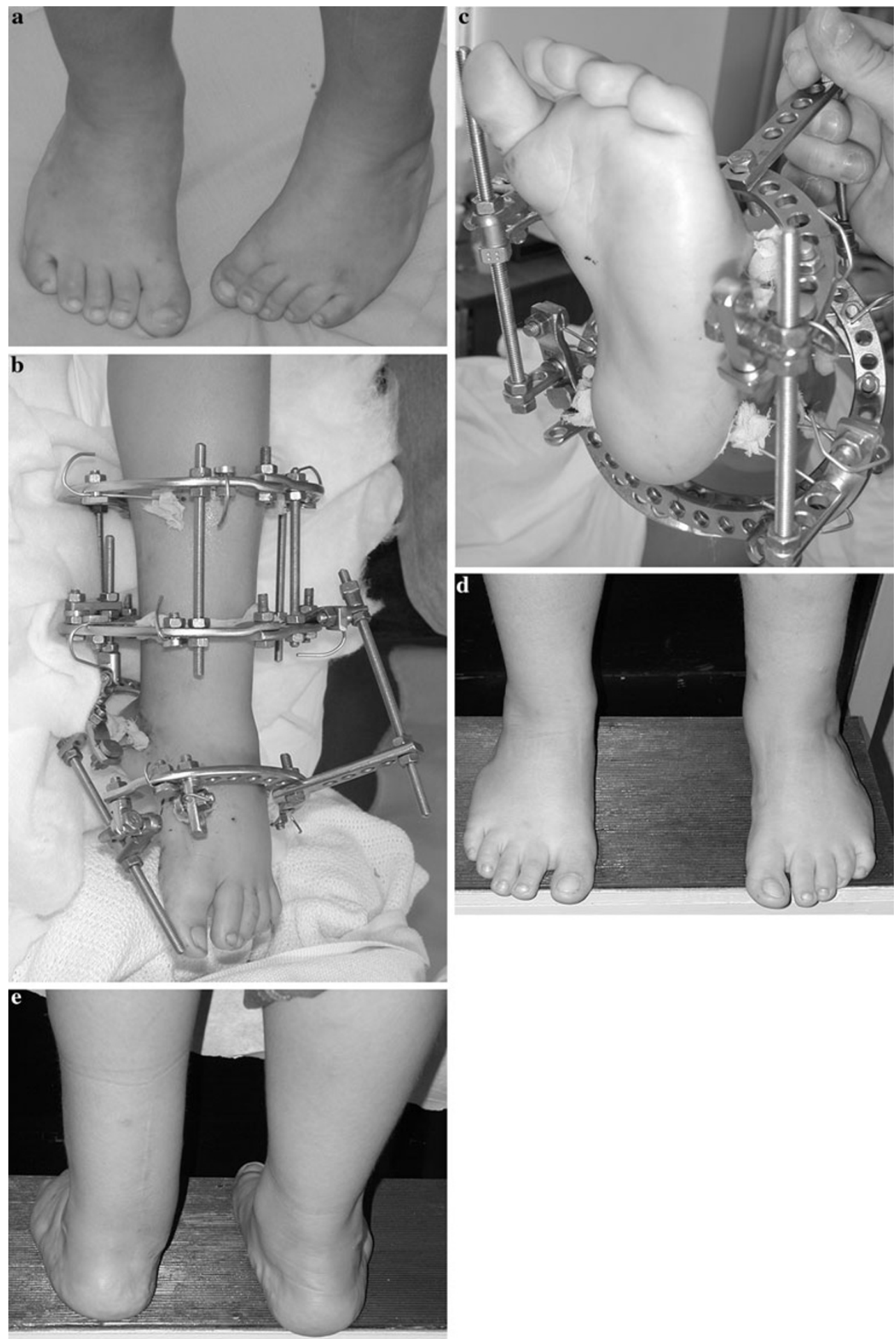

Fig. 1 a 6-year-old boy with relapsed left club foot. b, c The Ilizarov frame is applied as described in the text. d, e 3-year follow-up with a straight lateral border and heel valgus

manipulation under anaesthesia and bracing at the time of removal of the fixator. A first metatarsal bone fracture occurred during distraction in one patient and healed with angulation. It was addressed by percutaneous osteotomy and $\mathrm{K}$-wire fixation at the time of fixator removal.
Correction of the deformity was also associated with moderate to severe pain that was managed adequately with oral pain killers as previously described. Even so, a reduction in the correction rate was necessary in three patients. 

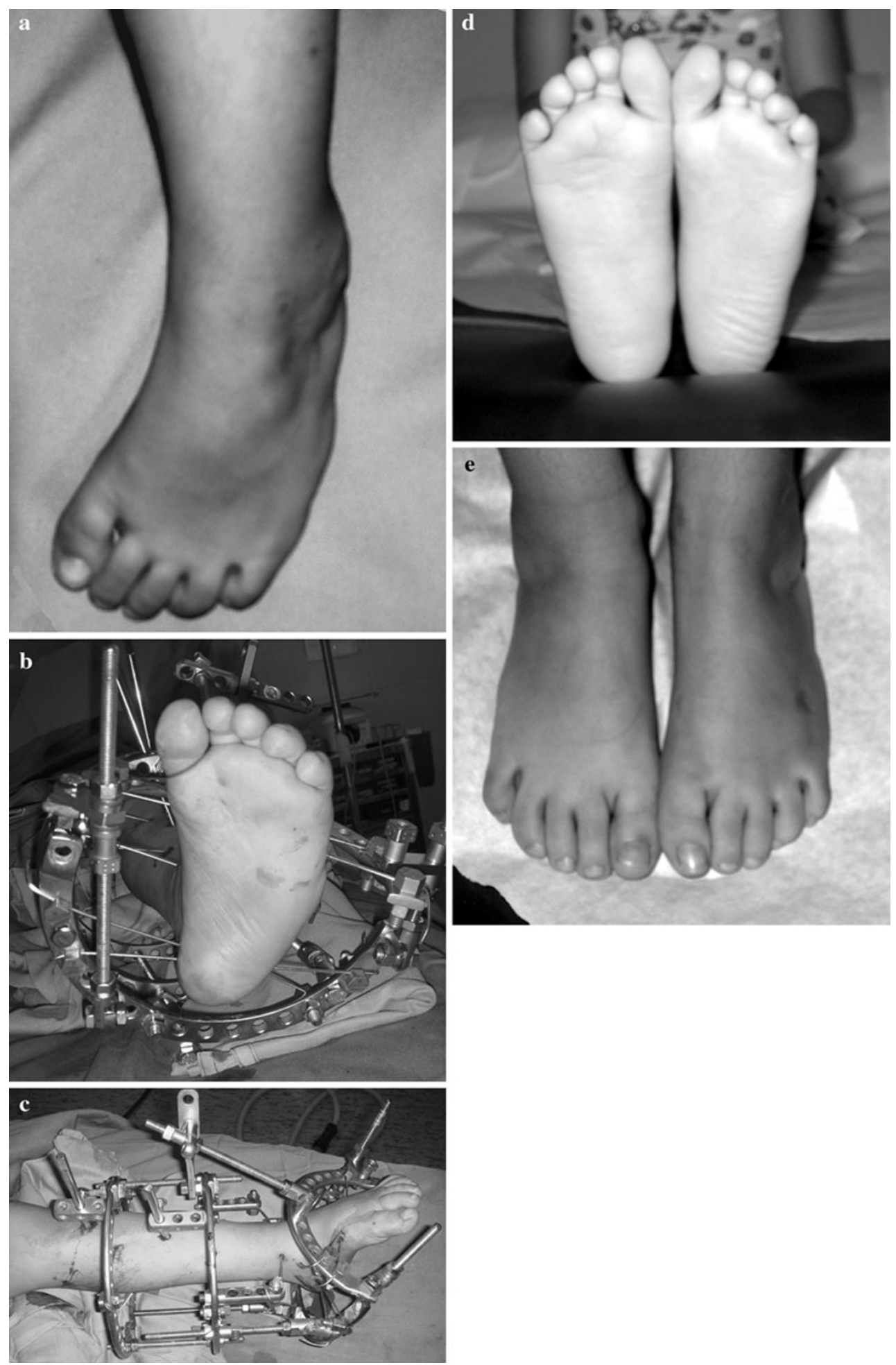

Fig. 2 a 5-year-old girl with relapsed left club foot. b, c The Ilizarov frame is applied as described in the text. d, e 2-year follow-up with a straight lateral border to the foot

\section{Discussion}

A main goal of treatment of the clubfoot deformity is to reduce or eliminate all the components of the deformity so that the patient has a functional, pain-free, normal-looking, plantigrade foot with good mobility, without calluses and not requiring modified shoes [3]. The toughest challenge of clubfoot correction by traditional surgical procedures is 
attempting an acute correction of all components of the deformity without causing major permanent damage to the foot. The problem is further complicated when faced with a relapsed club foot with scarring and stiffness secondary to previous surgeries. Atar et al. [4] emphasized that even the best revision surgeries do not result in a normal-looking foot. Limitations are inherent in the complex nature of these procedures and stiffness within the foot.

Despite the reported success of the Ilizarov method in the treatment of relapsed club foot [1, 5-8], the results of these publications have been presented according to the criteria that were either set by their respective authors or adopted from earlier publications. A uniform grading system that would enable paediatric orthopaedic surgeons to objectify outcomes in clubfoot treatment has been lacking. More than a dozen systems for evaluating the outcome of clubfoot treatment have been reported. Outcome analysis in club foot has traditionally been difficult because of different starting points for the analysis, a lack of standardization in deformity description, a variety of surgical techniques, various patient-based evaluations and poor agreement on the criteria which constitute a good result [9]. For instance, many of the satisfied patients in the series by Wallander would have had their outcomes rated as fair or poor if analysed according to the criteria set by Lehman et al. [5].

A closer look at the objective variables that are included in scoring systems in evaluating the outcome of clubfoot treatment [10] shows that they may be divided into the following: (a) criteria that evaluate the structure and appearance of the foot, (b) criteria that evaluate the function of the foot and (c) criteria that evaluate the tarsal relationships on radiographs. The International Clubfoot Study Group (ICFSG) recently published an evaluation system that takes all the above-mentioned criteria into account [2]. The interobserver and intraobserver reliability of this outcome tool was tested by Celebi et al. [11]. They found that interobserver reliability for all subgroup evaluations was $90 \%$ or over. Good intraobserver reliability was also found especially in the morphology and the functional evaluation subgroups. More recently, Prem et al. [8] reviewed 19 feet, using the Ilizarov fixator for an average of 4 months with a follow-up of at least 5 years. The results were good to excellent in 14 out of the 19 feet according to the International Clubfoot Study Group score. However, only the postoperative scores were recorded due to the retrospective nature of their study.

In our review, we have utilized the ICFSG system preoperatively and postoperatively in order to present a current and objective assessment that can be used for future comparison against studies using the same system. This score improved by an average of 25 points (13-38) per patient after treatment. Moreover, this score did not deteriorate over the period of the study except in two patients who had recurrence of the deformity within 6 months of removal of the fixator. At the latest follow-up, seven out of nine feet were classified as having good to excellent outcomes.

The ICFSG score is a global evaluation of all aspects of foot deformity. Its application to the monitoring of clubfoot treatment allows us to understand better the effects of the deformity correction and the limitations of any therapeutic approach. Closed distraction is very powerful in correcting the clubfoot deformity and in restoring a near normal foot shape, as was indicated in the morphological scores. Internal rotation is the only component that we were not able to correct by closed distraction. Two relapses were seen, one was in an arthrogrypotic foot and the second in a patient non-compliant with the bracing protocol. Both occurred within 6 months after removal of the fixator.

The functional evaluation has three main components. The first is the passive motion of foot and ankle joints. Stiffness of the small joints in the foot and ankle is a major concern after open surgical releases. It has been reported that closed distraction does not lead to further loss of motion in the foot and ankle joints [5]. In our small series, the ankle joint was fixed in equinus in all patients with a limited arc of motion of around 20 degrees preoperatively. After distraction treatment, the arc of motion of the ankle improved to an average of 40 degrees. This beneficial effect was not seen in the subtalar and the midtarsal joints. However, the three patients who had a flexible foot before treatment did not lose their mobility after surgery.

Muscle function is the second component of the functional evaluation. In our series, there was no added weakness of the muscles of the ankle and toes after treatment. Closed distraction preserves the integrity of the musculotendinous units and improves the biomechanical leverage of the muscles secondary to the improvement in both the position of the foot and the arc of motion of the ankle joint. These improvements in morphology, joint mobility and muscle function translate into better gait, a decrease in pain, normal shoe wear and subsequently greater participation in activities such as running and jumping.

Radiologically, a flat top talus was present in 6 feet and was the most common persistent anomaly in our series. The radiologic findings correlated well with the correction in the morphology of the treated feet; the majority of the radiographic parameters were corrected to normal values in all but the two patients who had a relapse of the deformity. This contradicts previously published radiographic results found in club feet treated with the Ponseti technique where there was a residual deformity in the hindfoot compensated by lateral displacement and angulation of the cuneiforms with respect to the navicular [12]. The most plausible explanation is that distraction treatment does not follow the 
same principles of Ponseti treatment. The calcaneum is well fixed and is not allowed to rotate freely under the talus in conjunction with midfoot abduction. Instead, it is forced to move into valgus and neutral dorsiflexion while both the midfoot and forefoot are being abducted through an independent mechanism created by the distraction rods. We believe that distraction with an external fixator is more potent than serial casting in restoring normal bony anatomy, although the overall morphology and the function of the foot are also addressed well by the latter method.

Our overall success rate using closed distraction is not significantly better than the reported results from open surgery [4, 13]. However, the reported complications with open releases may be devastating, in particular wound dehiscence. Wound closure, after acute correction by open release surgery, may require advancement flaps (myocutaneous or fasciocutaneous) or preoperative creation of extra skin by tissue expanders [13]. Even when the skin is closed primarily, the foot is casted in an under-corrected position for weeks to allow healing of the wound [4]. Recently, Nogueira et al. published their series of treating relapsed club feet by the Ponseti serial casting method with satisfactory results. Correction was obtained in $89 \%$ of cases but $14 \%$ relapsed later [14]. The technique is less invasive and less painful. However, it requires a foot abduction brace for at least 1 year. This type of bracing is tolerated better in toddlers but difficult to use with an older age group. The main advantage remains in the reported gain in the subtalar joint motion.

There are three points of weakness in this study. The first is the small number of patients enrolled in our series; the second is the treatment of both feet in one arthrogrypotic patient (possibly representing a different pathology); and the third is the relatively short-term follow-up. However, this study is a prospective observational series performed along preset guidelines, and the surgery performed by one surgeon using the same surgical technique in all patients. The use of ICFSG score preoperatively and on follow-up added to the objectivity of this study.

In conclusion, closed distraction treatment for relapse in a previously treated club foot carries an acceptable rate of success comparable to surgical release and serial casting. This method obviates the need for open extended releases, carries a low risk for major complications and corrects the anatomic and radiographic anomalies as well as improves the function. Postoperative bracing and close follow-up are mandatory to detect early recurrences and prevent significant relapses.

Open Access This article is distributed under the terms of the Creative Commons Attribution Noncommercial License which permits any noncommercial use, distribution, and reproduction in any medium, provided the original author(s) and source are credited.

\section{References}

1. Bradish CF, Noor S (2000) The Ilizarov method in the management of relapsed club feet. J Bone Joint Surg Br 82:387-391

2. Bensahel H, Kuo K, Duhaime M (2003) The International Clubfoot Study Group. Outcome and evaluation of the treatment of clubfoot: the international language of clubfoot. J Pediatr Orthop B 12:269-271

3. Ponseti I (1996) Treatment. In: Ponseti I (ed) Congenital clubfoot. Fundamentals of treatment. Oxford medical publications. Oxford University Press Inc, New York, pp 61-97

4. Atar D, Lehman WH, Grant AD, Strongwater AM (1992) Revision surgery in clubfeet. Clin Orthop 283:223-230

5. Wallander H, Tjernstrom B (1996) Correction of persistent clubfoot deformities with Ilizarov external fixator. Experience in 10 previously operated feet followed for $2-5$ years. Acta Orthop Scand 67:283-287

6. El Barbary H, Abdel Ghani H, Hegazy M (2004) Correction of relapsed or neglected clubfoot using a simple Ilizarov frame. Int Orthop 28:183-186

7. Utukuri MM, Ramachandran M, Hartley J, Hill RA (2006) Patient based outcomes after Ilizarov surgery in resistant clubfeet. J Pediatr Orthop B 15:278-284

8. Prem H et al (2007) Soft tissue Ilizarov correction of congenital talipes equinovarus-5 to 10 years postsurgery. J Pediatr Orthop 27(2):220-224

9. Buerk AA, Albert MC (2001) Advances in pediatric foot and ankle treatment. Curr Opin Orthop 12:437-442

10. Munshi S, Varghese RA, Joseph B (2006) Evaluation of outcome of treatment of congenital clubfoot. J Pediatr Orthop 26:664-672

11. Celebi L, Muratli HH, Akashin E, Yagmurlu MF, Bicimoglu A (2006) Bensahel et al. and International Clubfoot Study Group evaluation of treated clubfoot: assessment of interobserver and intraobserver reliability. J Pediatr Orthop B 15:34-36

12. Ponseti I, El-Khoury GY, Ippolito E, Weinstein S (1981) A radiographic study of skeletal deformities in treated clubfeet. Clin Orthop 160:30-42

13. Ettl V, Kirschner S, Krauspe R, Raab P (2009) Midterm results following revision surgery in clubfeet. Int Orthop 33:515-520

14. Nogueira MP, Ey Batlle AM, Alves CG (2009) Is it possible to treat recurrent clubfoot with the Ponseti technique after posteromedial release?: a preliminary study. Clin Orthop 467:1298-1305 